Gullierme Karam Corrêa LetTe ${ }^{1}$

Henri Augusto Korkes ${ }^{2}$

ArILDO de Toledo VIANA ${ }^{3}$

AleXANDRE PITORRI ${ }^{4}$

GReCy KenN ${ }^{5}$

NelsOn SASS ${ }^{6}$

\title{
Miomectomia em gestação de segundo trimestre: relato de caso
}

\author{
Myomectomy in the second trimester of pregnancy: case report
}

Artigo original

Palavras-chave

Mioma/cirurgia

Leiomioma

Gestação

Abdome agudo

Cesárea

Keywords

Myoma/surgery

Leiomyoma

Pregnancy

Abdomen, acute

Cesarean section

\section{Resumo}

Os leiomiomas uterinos caracterizam-se por doença benigna e são evidenciados em 2 a 3\% de todas as gestações normais. Destes, cerca de 10\% podem apresentar complicações durante a gravidez. Apresentamos um caso de paciente gestante que procurou o pronto-socorro obstétrico na $17^{a}$ semana, queixando-se de fortes dores, apresentava palpação abdominal dolorosa e descompressão brusca positiva. À ultrassonografia, apresentava nódulo de mioma medindo $9,1 \times 7,7 \mathrm{~cm}$, foi internada, medicada e devido à piora do quadro, submetida a laparotomia exploradora e miomectomia. $\bigcirc$ seguimento pré-natal se deu sem mais anormalidades, com resolução da gestação na $39^{a}$ semana. $\bigcirc$ recém-nascido pesou $3.315 \mathrm{~g} \mathrm{com} \mathrm{Apgar} 9$ e 10. Deve-se sempre tentar o tratamento clínico nesses casos, e intervenções cirúrgicas devem ser consideradas para casos selecionados, principalmente na impossibilidade de tratamentos conservadores ou quando o quadro clínico da paciente exige intervenção imediata. Neste caso, a miomectomia mostrou-se eficaz para complicações obstétricas materno-fetais.

\section{Abstract}

Uterine leiomyomas are characterized as a benign disease and are observed in 2 to $3 \%$ of all normal pregnancies. Out of these, about 10\% may present complications during pregnancy. We present a case of a pregnant patient sought emergency obstetric care at the 17th week, complaining of severe pain, presenting with painful abdominal palpation and sudden positive decompression. Ultrasonography revealed a myoma nodule measuring $9.1 \times 7.7 \mathrm{~cm}$; the patient was hospitalized and medicated, being also submitted to laparotomy and myomectomy due to worsening of her condition. Prenatal care revealed no further abnormalities, with resolution of gestation at 39 weeks. The newborn weighed 3,315 g, with Apgar scores of 9 and 10. In such cases, clinical treatment should always be attempted and surgery should be considered only in selected cases, mainly in the impossibility of conservative treatment or when the patient's clinical features require immediate intervention. In this case, myomectomy was effective against maternal-fetal obstetric complications.
Correspondência:

Guilherme Karam Corrêa Leite Rua Raul Pompéia, 1061/114 - Vila Pompéia CEP 02550-011 - São Paulo (SP), Brasil Tel. (11) 3567-1154 / 8356-3450 E-mail: guilhermekaram@uol.com.br

Recebido

28/9/09

Aceito com modificacões
Serviço de Obstetrícia do Hospital Maternidade Escola "Dr. Mario de Moraes Antenfelder Silva" da Maternidade Vila Nova Cachoeirinha - São Paulo (SP), Brasil.

'Médico Tocoginecologista do Hospital Municipal Maternidade Escola Dr. Mário de Moraes Altenfelder Silva - São Paulo (SP), Brasil.

2 Residente Nível R3 de Ginecologia e Obstetrícia do Hospital Municipal Maternidade Escola Dr. Mário de Moraes Altenfelder Silva São Paulo (SP), Brasil.

${ }^{3}$ Coordenador Técnico-Científico da Clínica Cirúrgica do Hospital Municipal Maternidade Escola Dr. Mário de Moraes Altenfelder Silva - São Paulo (SP), Brasil.

${ }^{4}$ Médico Tocoginecologista do Hospital Municipal Maternidade Escola Dr. Mário de Moraes Altenfelder Silva - São Paulo (SP), Brasil.

${ }^{5}$ Médica Tocoginecologista do Hospital Municipal Maternidade Escola Dr. Mário de Moraes Altenfelder Silva - São Paulo (SP), Brasil.

${ }^{\circ}$ Coordenador Chefe da Clínica Obstétrica do Hospital Municipal Maternidade Escola Dr. Mário de Moraes Altenfelder Silva - São Paulo (SP), Brasil. 


\section{Introdução}

O leiomioma uterino constitui condição benigna que pode acometer mulheres em idade fértil, principalmente a partir dos 30 anos (20-30\%). Podem estar presentes em exame de rotina em cerca de 2 a $3 \%$ das gestações e em cerca de $10 \%$ dos casos podem apresentar complicações ao longo do ciclo gravídico puerperal ${ }^{1,2}$. Ressalta-se um relativo aumento de sua prevalência na população obstétrica durante a última década, uma vez que as mulheres têm engravidado mais tardiamente ${ }^{3}$. Sendo assim, é possível encontrar na literatura descrições visando ao melhor entendimento das interações recíprocas entre a leiomiomatose e a gestação, tais como a identificação de fatores de riscos relacionados ao tamanho, número, características, localização e posição dos nódulos, e suas relações com a placenta ${ }^{2}$.

Enquanto na maioria dos casos os leiomiomas não estão associados a sintomas ou complicações durante a gestação ${ }^{1}$, em outros, esses nódulos têm sido associados a abortamento espontâneo, parto pré-termo, rotura prematura de membranas, sangramento anteparto, descolamento prematuro da placenta, apresentação anômala e maiores taxas de parto cesáreo ${ }^{4-6}$.

A dor abdominal é a principal queixa apresentada pela gestante com leiomioma e pode, em alguns casos, cursar com quadros álgicos significativos, inclusive com necessidade de internações. Na maioria das vezes, esse quadro álgico está relacionado com a degeneração vermelha, que ocorre em 5-8\% dos miomas na gestação ${ }^{4}$. Nestas circunstâncias, após a falha do tratamento medicamentoso, o tratamento cirúrgico com miomectomia deve ser considerado, ponderando-se os riscos associados a tal procedimento durante a gestação.

O presente estudo descreve caso raro de abdome agudo em gestação de segundo trimestre devido à degeneração miomatosa vermelha em que a miomectomia foi necessária. Os resultados do seguimento da gestação e do parto estão descritos e a literatura sobre o assunto foi revisada.

\section{Descrição do caso}

Primigesta, 43 anos, iniciou seguimento em setembro de 2007 no ambulatório de Pré-natal de Alto Risco do Hospital Municipal Maternidade Escola Dr. Mário de Moraes Altenfelder Silva (Vila Nova Cachoeirinha), São Paulo (SP). Foi encaminhada da Unidade Básica de Saúde (UBS) com ultrassonografia obstétrica de primeiro trimestre com dez semanas de gestação, que evidenciou um nódulo miomatoso em região cornual direita do útero medindo 6,2 x $5,9 \mathrm{~cm}$. A paciente foi assistida até o início do segundo trimestre sem intercorrências ou internações, apenas referindo dores abdominais ocasionais que melhoravam com o uso de analgésicos. $\mathrm{Na} 17^{\mathrm{a}}$ semana de gestação, procurou atendimento de urgência na referida Maternidade com queixa abdominal difusa que irradiava para fossa ilíaca direita havia dois dias. Na ocasião, estava em bom estado geral, corada, hidratada, afebril e eupneica. Ao exame clínico, tinha abdome semigloboso, altura uterina de 18 $\mathrm{cm}$, batimentos cardíacos fetais presentes e ausência de contrações uterinas. A palpação abdominal era dolorosa difusamente, mais intensa à direita, com defesa muscular e descompressão brusca positiva. Ao toque vaginal, o colo era posteriorizado, grosso e impérvio. Foi solicitado um hemograma completo que não apresentou alterações, e um exame de ultrassom obstétrico que evidenciou um nódulo hipoecoico, heterogêneo, anterolateral direito, sugestivo de leiomioma, predominantemente subseroso, medindo 9,1 x 7,7 cm (Figura 1).

A paciente foi internada para observação e medicada com analgésicos e anti-inflamatórios. Após aproximadamente 24 horas de evolução e apresentando piora importante do quadro doloroso, foi submetida à laparotomia exploradora. A cirurgia foi realizada sob raquianestesia e com incisão longitudinal. No inventário da cavidade, observou-se um leiomioma séssil em corno direito uterino de aproximadamente $10 \times 10 \mathrm{~cm}$ de diâmetro com sinais de necrose. Realizou-se a miomectomia com auxílio de bisturi elétrico e verificou-se que a lesão não comprometia o miométrio, sendo o leiomioma predominantemente subseroso. Feita a exérese do leiomioma, a sutura foi realizada com pontos separados em dois planos, utilizando-se fio absorvível de polyglactina (Vicryl $\left.{ }^{\circledR} 0\right)$. O ato operatório transcorreu sem intercorrências (Figura 2).

No pós-operatório imediato, a paciente foi submetida à tocólise profilática com terbutalina $\left(\right.$ Bricanyl $\left.^{\circledR}\right)$ por 48 horas e recebeu progesterona micronizada (Ultrogestan ${ }^{\circledR}$ ) na dose de $400 \mathrm{mg} /$ dia, sendo esta última suspensa durante a $34^{\mathrm{a}}$ semana de gestação. Não houve complicações pós-operatórias imediatas e a gestante recebeu alta no $4^{\circ}$ dia após a cirurgia.

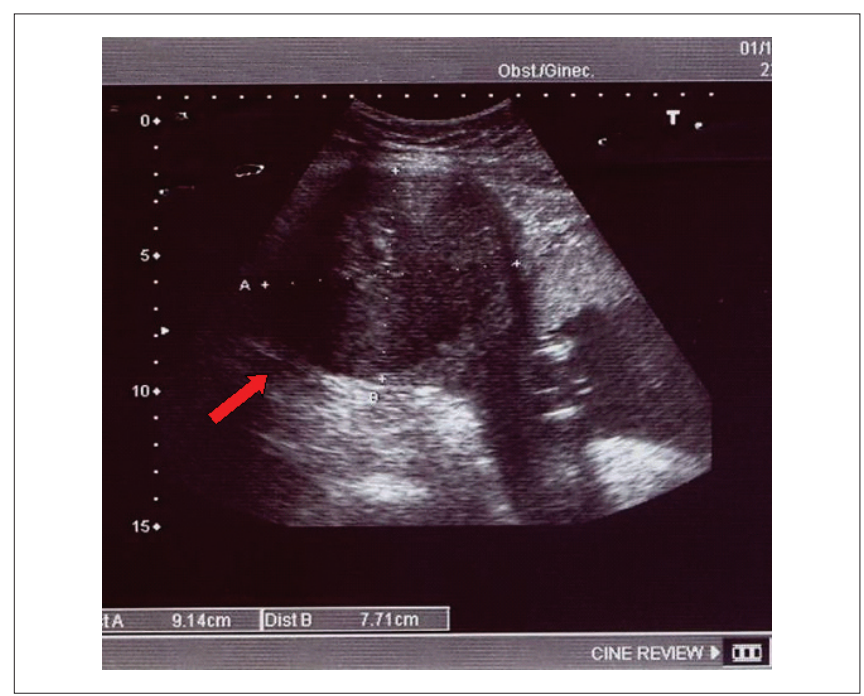

Figura 1 - Imagem ultrassonográfica de leiomioma, predominantemente subseroso, medindo 9,1 $1 \times 7,7 \mathrm{~cm}$. 

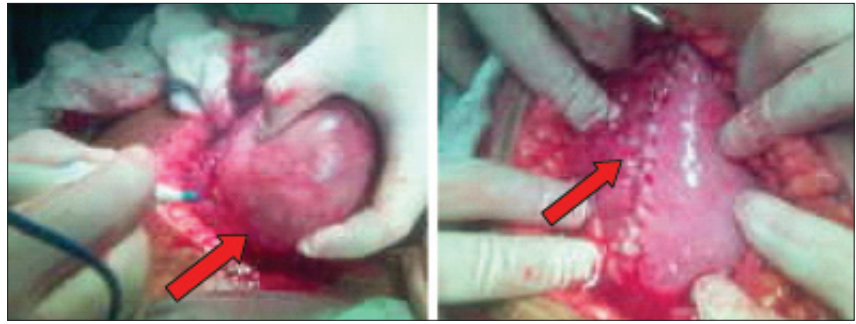

Figura 2 - Intraoperatório de laparotomia exploradora evidenciando o momento da realização da miomectomia com exérese de nódulo de mioma subseroso séssil de aproximadamente $10 \times 10 \mathrm{~cm}$. Em detalhe, aparência final da sutura uterina.

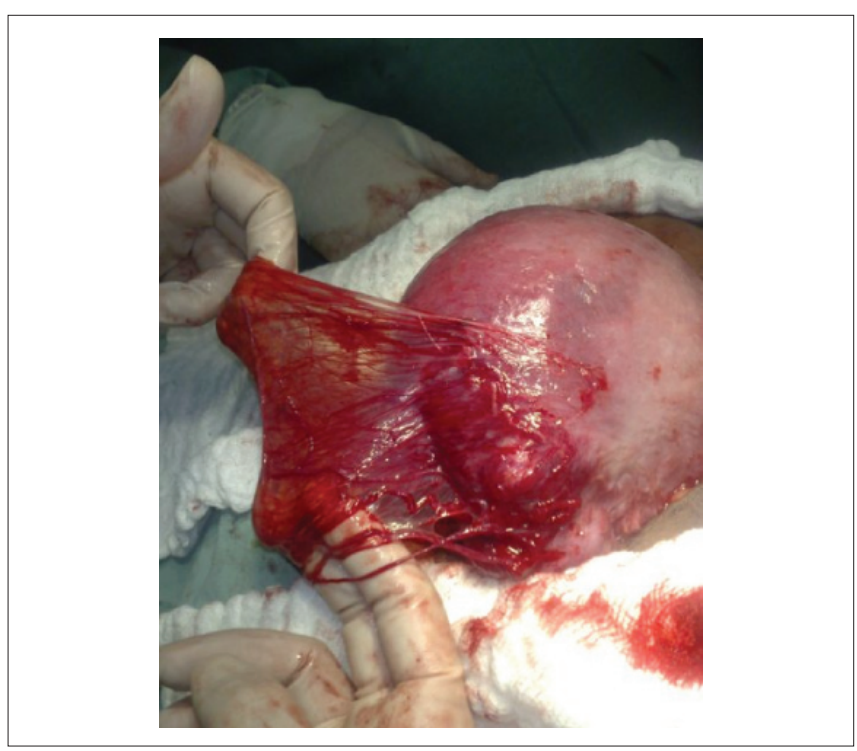

Figura 3 - Imagens do intraoperatório durante parto cesariano evidenciando cicatriz da miomectomia íntegra e com finas aderências epiploicas bastante vascularizadas.

O laudo anatomopatológico revelou leiomioma uterino com alterações degenerativas isquêmicas e necrose.

O seguimento pré-natal se deu sem mais anormalidades. A resolução da gestação foi feita de forma eletiva na $39^{\mathrm{a}}$ semana, com recém-nascido vivo, pesando $3.315 \mathrm{~g} \mathrm{e}$ com índices de Apgar 9 e 10 no primeiro e quinto minutos, respectivamente. Durante a cesariana, foi explorada a cicatriz da miomectomia que se apresentava íntegra, com finas aderências epiploicas bastante vascularizadas (Figura 3). A paciente recebeu alta juntamente com o recém-nascido no terceiro dia pós-operatório.

\section{Discussão}

A utilização de ultrassonografia durante a gestação tem permitido detectar precocemente os leiomiomas e acompanhar sua evolução, possibilitando a antecipação das possíveis complicações como torções e infartos. No que diz respeito ao tratamento de tais complicações, a miomectomia realizada durante a gestação caracteriza-se como prática controversa. Os melhores resultados mostravam taxas de sucesso em cerca de 2-16\% das gestações ${ }^{1,2,7,8}$, no entanto, estudos recentes não demonstraram maiores complicações em comparação a condutas conservadoras, o que proporciona maior segurança na sua manutenção após a conduta cirúrgica ${ }^{7-12}$.

Cerca de $10 \%$ das gestantes com leiomioma têm complicações relacionadas à presença deste tumor, as quais em sua maioria se relacionam à dor, usualmente observada no segundo trimestre, podendo ou não associar-se a náuseas, vômitos e febre ${ }^{13,14}$. Tal fato decorre principalmente da degeneração vermelha, também conhecida como degeneração carnosa ou necrobiose, devido ao infarto hemorrágico de parte do leiomioma por insuficiência vascular ${ }^{15}$. Outra possibilidade é a torção de um leiomioma pediculado que também pode resultar em quadro de abdome agudo ${ }^{4}$.

Além da dor recorrente, resistente à terapêutica medicamentosa usualmente realizada com analgésicos e anti-inflamatórios e da síndrome dolorosa aguda, alguns investigadores utilizaram outros critérios para indicação de miomectomia, selecionando pacientes elegíveis para o procedimento cirúrgico de forma eletiva, como: desconforto da paciente pelo crescimento tumoral, distância mínima de $5 \mathrm{~mm}$ da lesão para a cavidade endometrial ${ }^{1}$ e miomas de grande volume no segmento uterino ou deformando o sítio placentário ${ }^{8}$.

Salienta-se que tais situações devem ser discutidas com as pacientes, uma vez que o procedimento cirúrgico implica risco adicional de perda fetal; no entanto, sabemos que a prorrogação de uma cirurgia de urgência pode comprometer a sobrevida materna.

Tentando relacionar ainda fatores de maiores riscos, alguns autores sugerem que o descolamento prematuro de placenta é mais frequente quando há nódulos maiores que $200 \mathrm{~cm}^{3}$, submucosos ou implantados no sítio placentário ${ }^{2}$. Dor intensa relaciona-se com mioma de volume superior a $200 \mathrm{~cm}^{3}$, padrão ecoico heterogêneo e áreas císticas, sugerindo degeneração miomatosa ${ }^{2}$. Área cística no interior dos leiomiomas é relacionada com quadro álgico mais pronunciado, e este achado pode significar piomioma, ou seja, presença de conteúdo purulento, podendo, além de dor, apresentar febre ${ }^{15,16}$.

A ressonância magnética permite mensurar o tamanho e melhor avaliar as alterações arquiteturais degenerativas ${ }^{17}$, mas com uso limitado na gestação, sendo, na prática, menos útil no seguimento do volume do mioma do que a ultrassonografia. Neste ponto, a utilização do Doppler pode ajudar no manejo da patologia através do estudo da vasculatura uterino-placentária, sendo observado se as alterações do crescimento tumoral estão influenciando de alguma forma o fluxo placentário ${ }^{18}$, uma vez que o desenvolvimento do leiomioma pode de certa forma "roubar" grande parte deste fluxo, desviando o sangue para a nutrição tumoral.

Geralmente, o tratamento do mioma associado à gestação consiste na administração de analgésicos e repouso ${ }^{15}$. O uso de altas doses diárias de progesterona micronizada (300-600 
$\mathrm{mcg}$ ) parece favorecer a regressão dos sintomas em casos com suspeita de alterações degenerativas, pelo efeito no aumento do fluxo sanguíneo dos leiomiomas, em uma ou duas semanas $^{19}$. Em casos com manifestação clínica mais exuberante, analgesia peridural ou miomectomia são necessárias ${ }^{15}$.

É sabido que a miomectomia aumenta significativamente o número de cesáreas eletivas entre estas pacientes ${ }^{8,9,11,18}$. Porém, alguns autores afirmam que tanto a miomectomia histeroscópica quanto a laparotômica não impedem o trabalho de parto ou a administração de ocitócito9. Para outros, a frequência de abortamento após a miomectomia não difere significativamente das gestantes tratadas conservadoramente ${ }^{7,8}$.
Os casos de rotura uterina foram mais frequente em casos de cirurgia laparoscópica9,20,21. De maneira geral, a histerectomia na gestação é mais frequente em mulheres com mioma do que na ausência desta condição ${ }^{8}$.

Por fim, concluímos que a miomectomia é um procedimento a ser considerado durante a gestação em casos selecionados, principalmente na falha terapêutica medicamentosa da síndrome dolorosa da gestação. Orientamos, entretanto, sempre que possível, o acompanhamento conservador, relevando-se as condições clínicas da paciente e atentando para as alterações do leiomioma no seguimento ultrassonográfico gestacional.

\section{Referências}

1. Lolis DE, Kalantaridou SN, Makrydimas G, Sotiriadis A, Navrozoglou I, Zikopoulos K, et al. Successful myomectomy during pregnancy. Hum Reprod. 2003;18(8):1699-702.

2. Exacoustòs $C$, Rosati P. Ultrasound diagnosis of uterine myomas and complications in pregnancy. Obstet Gynecol. 1993;82(1): 97-101.

3. Bałoniak B, Słomko Z, Malewski Z, Drews K. The incidence of uterine leiomyomas in pregnancy and their influence upon its course. Ginekol Pol. 2002;73(4):260-5.

4. Phelan JP. Myomas and pregnancy. Obstet Gynecol Clin North Am. 1995;22(4):801-5.

5. Aydeniz B, Wallwiener D, Kocer C, Grischke EM, Diel IJ, Sohn C, et al. Significance of myoma-induced complications in pregnancy. A comparative analysis of pregnancy course with and without myoma involvement. Z Geburtshilfe Neonatol. 1998;202(4): 154-8.

6. Vergani P, Ghidini A, Strobelt N, Roncaglia N, Locatelli A, Lapinski $\mathrm{RH}$, et al. Do uterine leiomyomas influence pregnancy outcome? Am J Perinatol. 1994; 11 (5):356-8.

7. Bhatla N, Dash BB, Kriplani A, Agarwal N. Myomectomy during pregnancy: a feasible option. J Obstet Gynaecol Res. 2009;35(1):173-5.

8. Mollica G, Pittini L, Minganti E, Perri G, Pansini F. Elective uterine myomectomy in pregnant women. Clin Exp Obstet Gynecol. 1996;23(3): 168-72.

9. Kelly BA, Bright P, Mackenzie IZ. Does the surgical approach used for myomectomy influence the morbidity in subsequent pregnancy? J Obstet Gynaecol. 2008;28(1):77-81 .

10. Bonito M, Gulemì L, Basili R, Roselli D. Myomectomy during the first and second trimester of pregnancy. Clin Exp Obstet Gynecol. 2007;34(3): 149-50.
11. Celik C, Acar A, Ciçek N, Gezginc K, Akyürek C. Can myomectomy be performed during pregnancy? Gynecol Obstet Invest. 2002;53(2): 79-83.

12. Hasbargen U, Strauss A, Summerer-Moustaki M, Baretton G, Roth U, Kimmig R, et al. Myomectomy as a pregnancy-preserving option in the carefully selected patient. Fetal Diagn Ther. 2002;17(2):101-3.

13. Okonkwo JE, Udigwe GO. Myomectomia in pregnancy. J Obstet Gynecol. 2007;27(6):628-30.

14. Lanovette JM, Diamond MP. Pregnancy in women with myoma uteri. Infertil Reprod Med North Am. 1996;7(1):19-32.

15. Bozzine N. Leiomioma uterino e gravidez. In: Pompei LM, Sancovski M, Peixoto P. Leiomioma uterino. São Paulo: PlanMark; 2005. p. 177-84.

16. Królikowska B, Zmudzi ska E, Kobierski J, Makarewicz $\mathrm{H}$, Olszewski J. Co-existence of myoma uteri and pregnancy. Ginekol Pol. 2002;73(4):280-3

17. Okizuka H, Sugimura K, Takemori M, Obayashi C, Kitao M, Ishida T. MR detection of degenerating uterine leiomyomas. J Comput Assist Tomogr. 1993;17(5):760-6.

18. Gojnic M, Pervulov M, Petkovic S, Papic M, Jeremic K, Mostic $\mathrm{T}$. Indication of myomectomy during pregnancy from Doppler ultrasonography. Clin Exp Obstet Gynecol. 2004;31(3):197-8.

19. Hájek Z, Uhlír M. [Micronized progesterone in the treatment of imminent necrosis of a myoma during pregnancy. Ultrasound changes during treatment.] Ceska Gynekol. 1999;64(3): 189-92.

20. Hockstein S. Spontaneous uterine rupture in the early third trimester after laparoscopically assisted myomectomy. A case report. J Reprod Med. 2000;45(2):139-41.

21. Wong L, Ching TW, Kok TL, Koon TH. Spontaneous hemoperitoneum from a uterine leiomyoma in pregnancy. Acta Obstet Gynecol Scand. 2005;84(12):1208-9. 